\title{
Aceites vegetales sobre ácidos grasos y producción de metano in vitro en vacas lecheras ${ }^{1}$
}

\section{Vegetable oils on in vitro fatty acids and methane production in dairy cows}

\author{
Esperanza Prieto-Manrique², Julio Ernesto Vargas-Sánchez ${ }^{3}$,Joaquín Angulo-Arizala ${ }^{4}$, \\ Liliana Mahecha-Ledesma ${ }^{4}$
}

\section{RESUMEN}

El objetivo de este trabajo fue evaluar el efecto de la adición de aceites vegetales en dietas representativas de vacas lecheras bajo pastoreo, sobre los ácidos grasos, fermentación ruminal y producción de metano in vitro. La investigación se desarrolló en el laboratorio NUTRILABGRICA, perteneciente a la Facultad de Ciencias Agrarias, Universidad de Antioquia, Medellín, Colombia, en agosto del 2013. Se utilizó la técnica de producción de gas in vitro. En cuatro dietas (dos de monocultivo en Cynodon plectostachyus y dos de sistema silvopastoril intensivo con Leucaena leucocephala), se evaluó la adición de tres aceites vegetales (girasol, lino y palma) a nivel del 2 y $4 \%$ de la MS, en un diseño completamente al azar con arreglo factorial de $4 \times 3 \times 2$. En todas las dietas, la suplementación con aceite de girasol aumentó el contenido de ácido linoleico $(\mathrm{C} 18: 2$ c9,12), ácido linoleico conjugado (ALC-c9t11) y ácido transvaccénico (ATV, C18:1 t11) después de la fermentación ( $\mathrm{p}<0,0001)$, redujo en 2,4 y $0,9 \%$ la digestibilidad de la MS con respecto a aceite de palma $(\mathrm{p}<0,05)$ y lino $(\mathrm{p}>0,05)$. Ningún aceite afectó la cinética de fermentación, $\mathrm{pH}$, total de ácidos grasos volátiles (AGV), proporción de $\mathrm{AGV}$, ni la producción de metano.

Palabras clave: ácido linoleico conjugado, ácido transvaccénico, fermentación ruminal, ganado lechero en pastoreo, sistema silvopastoril.

\begin{abstract}
The aim of this study was to evaluate the effect of the addition of vegetable oils to representative diets of dairy cows under grazing, on the in vitro fatty acids, ruminal fermentation and methane production. The research was conducted in the laboratory NUTRILAB-GRICA, belonging to the Faculty of Agricultural Sciences, University of Antioquia, Medellin, Colombia, in August 2013. The technique of in vitro gas production was used. In four diets (two Cynodon plectostachyus monoculture and two intensive silvopastoral system with Leucaena leucocephala), the addition of three vegetable oils (sunflower, linseed and palm) at 2 and $4 \%$ of MS was assessed using a factorial design completely random $4 \times 3 \times 2$. In all diets, supplementation with sunflower oil increased the content of linoleic acid (C18: 2 c9, 12), conjugated linoleic acid (CLA-c9t11) and transvaccénico acid (ATV, C18:1 t11) after fermentation $(\mathrm{p}<0.0001)$, decreased by 2,4 and $0.9 \%$ DM digestibility regarding palm oil $(\mathrm{p}<0.05)$ and linseed oil $(\mathrm{p}>0.05)$. No oil affected fermentation kinetics, $\mathrm{pH}$, total of volatile fatty acid (VFA), VFA ratio or methane production.
\end{abstract}

Keywords: conjugated linoleic acid, trans vaccenic acid, ruminal fermentation, dairy cattle under grazing, silvopastoral system.

\footnotetext{
1 Recibido: 26 de noviembre, 2015. Aceptado: 1 de marzo, 2016. Este trabajo forma parte de un proyecto de investigación financiado por COLCIENCIAS - Colombia y es parte de la tesis doctoral del primer autor.

2 Universidad de Sucre, Facultad de Ciencias Agropecuarias, Carrera 28 N 5-267, Barrio Puerta Roja, Sincelejo-Sucre, Colombia. esperanza. prieto@unisucre.edu.co

3 Universidad de Caldas, Facultad de Ciencias Agropecuarias, Departamento Sistemas de Producción. Calle 65 N ${ }^{\circ} 26-10$, Manizales - Caldas, Colombia.jvargas@ucaldas.edu.co

4 Universidad de Antioquia, Facultad de Ciencias Agrarias, Grupo de Investigación en Ciencias Animales (GRICA), sede Robledo, Carrera 75 N ${ }^{\circ}$ 65-87 bloque 46, Medellín - Antioquia, Colombia. joaquin.angulo@udea.edu.co, Liliana.mahecha@udea.edu.co (autor para correspondencia).
} 


\section{INTRODUCCIÓN}

La producción de leche en Colombia es de gran importancia para la economía del país. La política para el sector lácteo colombiano busca mejorar la productividad de este sector, mediante el desarrollo de ventajas competitivas (CONPES, 2010). Razón por la cual, se busca el aumento de la oferta de productos lácteos funcionales y la producción amigable con el ambiente y socialmente responsable (CNL, 2010).

Como alimentos funcionales se consideran aquellos alimentos y/o componentes de este que poseen propiedades adicionales sobre la salud, que superen el beneficio clásico de un aporte de nutrientes (Milner, 1999). Los ácidos grasos insaturados como el ácido linoleico conjugado C18:2 c9t11 (ALCc9t11) o ruménico, el ácido transvaccénico C18:1 t11 (ATV) y algunos ácidos grasos de cadena larga (AGCL) $n$-3 de la leche bovina, se relacionan con beneficios para la salud humana (Harris, 2008). El interés que ha suscitado el ALC-c9t11 proviene de sus potenciales beneficios, observados experimentalmente sobre todo en modelos animales, como agente antiarteriosclerótico, antiinflamatorio, antidiabético y sobre todo, anti carcinogénico (inhibe la mutagénesis), así como potenciador del sistema inmune (Belury, 2002; Khanal, 2004; Pariza, 2004; Weiss et al., 2004a, 2004b; Shingfield et al., 2008). De acuerdo con lo anterior, ALC-c9t11 puede tener varios beneficios potenciales en los seres humanos.

En la leche bovina ALC y ATV resultan del consumo de ácidos grasos (AG) insaturados y de la extensión de la biohidrogenación ruminal (BHR); mientras que los AG $n-3$ provienen de la dieta, su presencia en la leche depende de su capacidad para escapar a la biohidrogenación ruminal (Chilliard et al., 2003; Palmquist, 2007). La magnitud de las cantidades presentes en la leche está determinada, principalmente, por factores dietarios (Palmquist et al., 2005). Se estima que más del 74\% de ALC-c9t11 en la grasa de la leche se sintetiza en la glándula mamaria, mediante la actividad de la enzima delta-9 desaturasa, a partir de ATV (Bichi et al., 2012); por lo tanto, es necesario aumentar el flujo de ATV desde el rumen para aumentar el contenido de ALC-c9t11 en la leche.

Uno de los mayores contaminantes del ambiente es el metano, gas de efecto invernadero que tiene un potencial de calentamiento veintiún veces mayor que el dióxido de carbono (IPCC, 2007). A la producción agrícola se le atribuye $40 \%$ de la producción de metano originado en actividades humanas. La producción de metano entérica, principalmente de la ganadería, constituye la mayor fuente individual y alcanza de 15 a $20 \%$ de la producción global de gases efecto invernadero de origen antrópico (Lassey et al., 1997; Moss et al., 2000; Sheehle y Kruger, 2006). La producción ruminal de metano constituye una pérdida de eficiencia nutricional que alcanza de 6 a $8 \%$ de la energía bruta consumida, pero puede incluso llegar hasta $12 \%$ de la misma (Johnson y Johnson, 1995). Razón por la cual, la búsqueda de tecnologías que permitan reducir la producción de metano en condiciones comerciales es tema de estudio.

En Colombia, el 45\% de la leche se produce en lechería especializada y el 55\% bajo el sistema doble propósito (CONPES, 2010); en su expresión tradicional se manejan bajo pastoreo con una base forrajera de solo gramíneas, sin o con suplementación de alimentos concentrados, con el fin de cubrir los requerimientos nutricionales de las vacas. No obstante, otros productores utilizan pastoreo en sistemas con una base forrajera silvopastoril, que permite aumentar la oferta de forraje, en particular durante el periodo seco, mejorar la calidad de la dieta a lo largo del año, la conservación y el reciclaje de nutrientes (Murgueitio, 1999; Pagiola et al., 2005, 2007). Hay evidencia de que metabolitos secundarios, como los taninos, presentes en plantas forrajeras no gramíneas, pueden afectar la biohidrogenación ruminal (Khiaosa-Ard et al., 2009) y la producción de metano (Jayanegara et al., 2011; Tan et al., 2011).

La suplementación con lípidos permite aumentar ALC-c9t11 y reducir la producción de metano. La suplementación con aceites de origen vegetal sugiere que aquellos con contenidos más altos de ácidos linoleico y linolénico (como los procedentes de semillas de soja, algodón, girasol, lino, cártamo y colza), son los más idóneos para aumentar el ALC-c9t11 en leche (Stanton et al., 2003; Khanal y Olson, 2004), este efecto es lineal ante el agregado de cantidades crecientes de aceite a la ración (hasta 3-4\% de la MS) (Chilliard et al., 2007). Además, se ha comprobado que aquellos más ricos en ácido linoleico (girasol, soja) son los más efectivos (Kelly et al., 1998; Dhiman et al., 2000; Lock y Garnsworthy, 2002; Collomb et al., 2004; Hervás et al., 2006; Shingfield et 
al., 2006), aunque la respuesta puede variar de acuerdo a la relación forraje:concentrado (Bauman y Griinari, 2001) y a la presencia de taninos (Vasta et al., 2009; Minieri et al., 2014). Así mismo, la suplementación con lípidos reduce la emisión de metano, a través de diferentes mecanismos que incluyen la disminución de la cantidad de materia orgánica fermentada en el rumen, disminución de la actividad de las bacterias metanogénicas y del número de protozoos, y mayor uso de hidrógeno durante el proceso de biohidrogenación (Johnson y Johnson, 1995; Beauchemin et al., 2009). La respuesta inhibitoria de las grasas en la producción de metano depende de la concentración, el tipo, la composición de ácidos grasos de las grasas y la composición nutricional de las dietas (Machmüller, 2006; Beauchemin et al., 2008).

El desarrollo de estrategias de suplementación en la ganadería colombiana con ácidos grasos poliinsaturados que permitan aumentar los ácidos grasos benéficos en la leche y disminuir la emisión de metano, exige un previo conocimiento del efecto de diferentes fuentes de ácidos grasos poliinsaturados bajo condiciones de alimentación específicas del país; estas fuentes varían de acuerdo con el sistema de producción y a los procesos de fermentación ruminal que se generan en cada uno de los animales, condiciones que deben ser consideradas para seleccionar la mejor opción, para posteriormente evaluarla en campo.

El objetivo de este trabajo fue evaluar el efecto de la adición de aceites vegetales en dietas representativas de vacas lecheras bajo pastoreo, sobre los ácidos grasos, fermentación ruminal y producción de metano in vitro.

\section{MATERIALES Y MÉTODOS}

\section{Localización}

Este trabajo fue desarrollado en el laboratorio NUTRILAB-GRICA, perteneciente a la Facultad de Ciencias Agrarias, Universidad de Antioquia, Medellín, Colombia, durante el mes de agosto del 2013.

\section{Diseño experimental}

El estudio se realizó mediante la técnica de producción de gas in vitro (Menke y Steingass, 1988; Theodorou et al., 1994), utilizando un diseño completamente al azar con arreglo factorial de tratamientos $4 \times 3 \times 2$ (cuatro dietas $\mathrm{x}$ tres aceites $\mathrm{x}$ dos niveles de inclusión de aceite). Se evaluaron cuatro dietas representativas de ganaderías de los sistemas lechería tropical (LT) y doble propósito (DP), que pastorean en solo gramíneas de estrella (Cynodon plectostachyus) y en sistemas silvopastoriles intensivos (SSPi) en estrella (Cynodon plectostachyus) o guinea (Megathyrsus maximus cv. Tanzania) con leucaena (Leucaena leucocephala), que fueron identificadas en un estudio previo de caracterización (Prieto-Manrique, 2015). Las dietas se definieron de la siguiente forma:

1. Pasto estrella $65 \%+$ concentrado $35 \%$. Esta dieta corresponde a la utilizada en sistemas de lechería tropical con monocultivo de pasto estrella y en adelante se llamará lechería tropical (LT).

2. Pasto estrella $59 \%$ + leucaena $15 \%+$ concentrado $26 \%$. Esta dieta corresponde a la utilizada en sistemas de lechería tropical con sistema silvopastoril intensivo y en adelante se llamará lechería tropical SSPi (LTSSPi).

3. Pasto estrella $80 \%+$ concentrado 20\%. Esta dieta corresponde a la utilizada en sistemas doble propósito con monocultivo de pasto estrella y en adeante se llamará doble propósito (DP).

4. Pasto guinea $84 \%$ + leucaena $16 \%$. Esta dieta corresponde a la utilizada en sistemas doble propósito con sistema silvopastoril intensivo y en adelante se llamará doble propósito SSPi (DPSSPi).

A las dietas se les adicionaron tres aceites comerciales: palma, girasol y lino (Cuadro 1), utilizando dos niveles correspondientes al $2(10 \mathrm{mg}$ de aceite $/ 500 \mathrm{mg}$ de sustrato) y $4 \%$ (20 $\mathrm{mg}$ de aceite $/ 500$ mg de sustrato) de la dieta en base seca.

Las muestras de forraje (pasto estrella, pasto guinea y leucaena) y de concentrado empleadas provenían de una sola finca representativa, manejada bajo sistema silvopastoril intensivo. Los forrajes tenían una edad de rebrote de cuarenta días y su cosecha se realizó durante la época de lluvias, simulando el pastoreo y ramoneo que realizan los animales, con base en estudios de comportamiento previos realizados en estos sistemas (Mahecha et al., 2000). Los forrajes se colocaron en estufa a $60{ }^{\circ} \mathrm{C}$ durante $48 \mathrm{~h}$ y luego fueron molidos, al igual que el concentrado, utilizando una criba de $1 \mathrm{~mm}$. 
Cuadro 1. Ácidos grasos de los aceites utilizados en la evaluación in vitro. Medellín, Colombia. Agosto, 2013.

Table 1. Fatty acids of the oils used in the in vitro evaluation. Medellin, Colombia. August, 2013.

\begin{tabular}{lccc}
\hline Ácido graso & $\begin{array}{c}\text { Aceite de } \\
\text { girasol }\end{array}$ & $\begin{array}{c}\text { Aceite de } \\
\text { lino }\end{array}$ & $\begin{array}{c}\text { Aceite de } \\
\text { palma }\end{array}$ \\
\hline & g AG/100g AG totales & \\
\hline C16:0 & 7,66 & 6,50 & 46,57 \\
C17:1 c10 & 1,80 & 0,04 & 2,78 \\
C18:0 & 4,22 & 3,56 & 4,33 \\
C18:1 c9 & 28,23 & 21,48 & 34,69 \\
C18:2 c9,12 & 55,95 & 18,66 & 9,58 \\
C18:3 c9,12,15 & 0,22 & 48,78 & 0,24 \\
\hline
\end{tabular}

\section{Composición química y perfil de ácidos grasos de los tratamientos}

Se tomó una muestra de cada tratamiento (mezcla de dieta más aceite), para determinar la composición química y el perfil de ácidos grasos (Cuadros 2 y 3). La composición química se realizó mediante las técnicas analíticas convencionales de la AOAC (1999) (materia seca método ID 934.01, cenizas método ID 942.05, proteína bruta método ID 984.13, grasa y fibra detergente ácido método ID 973.18) y los descritos por Van Soest et al. (1991) para los análisis de fibra detergente neutro y fibra detergente ácido.

El perfil de ácidos grasos de los aceites (Cuadro 1) y de los tratamientos (Cuadros 2 y 3), se obtuvo mediante el método de cromatografía de gases acoplado a espectrometría de masas, siguiendo la metodología propuesta por Tequin-Ocampo (2014), tanto para la extracción-derivatización como para el análisis cromatográfico en sí mismo. Se utilizó un cromatógrafo de gases acoplado a espectrómetro de masas, con automuestreador.

Las condiciones cromatográficas fueron las siguientes: 1) fase móvil: gas transportador helio, flujo de columna $1 \mathrm{ml} / \mathrm{min}$, velocidad lineal $26 \mathrm{~cm} / \mathrm{seg}$; 2) inyector: temperatura $220^{\circ} \mathrm{C}$, volumen $0,2 \mathrm{ul}$, modo Splitless; 3) columna: modelo CP - Sil - 88, longitud $100 \mathrm{~m}$, diámetro interno $0,25 \mathrm{~mm}$, espesor de la película $0,2 \mathrm{ul}$; 4) rampa de temperatura: temperatura $150{ }^{\circ} \mathrm{C}$, tiempo de calentamiento tres minutos, rata
$15{ }^{\circ} \mathrm{C} / \mathrm{min}$; 5) detector: temperatura $250{ }^{\circ} \mathrm{C}$, flujo de $\mathrm{N}^{2} 10 \mathrm{ml} / \mathrm{min}$.

Los ácidos grasos fueron separados e identificados por comparación de los tiempos de retención con sus respectivos estándares y por comparación de la librería del equipo; se cuantificaron utilizando la curva de calibración de los estándares de ALC y sus isómeros, de ATV y los demás ácidos grasos. Se utilizó como estándar interno ácido nonadecanoico (C19:0). El porcentaje de cada AG fue calculado a partir de su concentración (ppm), determinada por cromatografía (Tequin-Ocampo, 2014).

Adicionalmente, se tomó una muestra de leucaena para la determinación de taninos, realizada de acuerdo con Makkar (2003). Con base en esta información y con la proporción de leucaena presente en las dietas de los sistemas silvopastoriles, se estimó la concentración de taninos (Cuadros 2 y 3 ).

\section{Preparación del medio}

La solución tampón se preparó un día antes del inicio del ensayo, de acuerdo con las recomendaciones de McDougall (1948).

\section{Colecta del inóculo}

En cada tratamiento se usaron tres inóculos, procedentes de tres novillos que consumían pastos Cynodon plectostachyus, Panicum máximum, Brachiaria mutica y Dichantium aristatum Benth. La colecta del líquido ruminal se hizo inmediatamente después del sacrificio, se filtró en paños de algodón y se almacenó en termos precalentados con agua a $40{ }^{\circ} \mathrm{C}$. En el laboratorio, el líquido ruminal de cada animal se filtró nuevamente y fue transferido a tres erlenmeyer (uno por cada animal), los cuales fueron saturados con $\mathrm{CO}_{2}$ y mantenidos en estufa a $39^{\circ} \mathrm{C}$, durante el tiempo que demoró la inoculación.

\section{Determinación de la cinética de fermentación y la degradación in vitro de la materia seca}

La cinética de fermentación se determinó mediante la técnica de producción de gas in vitro (Menke y Steingass, 1988; Theodorou et al., 1994), modificada por Posada et al. (2006). Se usaron botellas de $100 \mathrm{ml}$ de capacidad, en las que se colocó 0,5 g de sustrato 
Cuadro 2. Composición nutricional y perfil de ácidos grasos de las dietas representativas de los sistemas lechería tropical, con adición de diferentes aceites a nivel del 2\% y 4\% de la MS utilizadas en la evaluación in vitro antes de la incubación por veinticuatro horas. Medellín, Colombia. Agosto, 2013.

Table 2. Nutritional composition and fatty acid profile of the representative diets of tropical dairy systems with different oil addition to level of $2 \%$ and $4 \%$ of the DM used in the in vitro evaluation before incubation for twenty four hours. Medellin, Colombia. August, 2013.

\begin{tabular}{|c|c|c|c|c|c|c|c|c|c|c|c|c|}
\hline \multirow{4}{*}{$\begin{array}{c}\text { Dieta/Sistema } \\
\text { Aceite } \\
\text { Nivel }\end{array}$} & \multirow{2}{*}{\multicolumn{6}{|c|}{$\begin{array}{c}\text { Lechería tropical } \\
\text { Estrella } 65 \%+\text { concentrado } 35 \%\end{array}$}} & \multirow{2}{*}{\multicolumn{6}{|c|}{$\begin{array}{l}\text { Lechería tropical SSPi } \\
\text { strella } 59 \%+\text { leucaena } 15 \%+ \\
\text { concentrado } 26 \%\end{array}$}} \\
\hline & & & & & & & & & & & & \\
\hline & \multicolumn{2}{|c|}{ Girasol } & \multicolumn{2}{|c|}{ Lino } & \multicolumn{2}{|c|}{ Palma } & \multicolumn{2}{|c|}{ Girasol } & \multicolumn{2}{|c|}{ Lino } & \multicolumn{2}{|c|}{ Palma } \\
\hline & $2 \%$ & $4 \%$ & $2 \%$ & $4 \%$ & $2 \%$ & $4 \%$ & $2 \%$ & $4 \%$ & $2 \%$ & $4 \%$ & $2 \%$ & $4 \%$ \\
\hline$\%$ grasa & 5,7 & 7,6 & 5,6 & 6,4 & 5,1 & 7,1 & 4,7 & 6,6 & 4,8 & 6,9 & 4,9 & 6,4 \\
\hline \% proteína & 12,2 & 12,4 & 12,8 & 12,1 & 11,8 & 12,0 & 13,8 & 12,8 & 14,8 & 14,1 & 14,7 & 13,8 \\
\hline$\% \mathrm{FDN}$ & 57,6 & 55,6 & 58,8 & 60,7 & 61,7 & 57,7 & 57,1 & 56,0 & 55,9 & 55,5 & 56,6 & 55,5 \\
\hline$\%$ FDA & 25,5 & 25,1 & 25,8 & 26,7 & 26,8 & 25,5 & 24,9 & 24,3 & 24,8 & 24,2 & 25,1 & 24,2 \\
\hline$\%$ cenizas & 10,2 & 10,0 & 10,0 & 10,0 & 9,8 & 9,9 & 9,6 & 9,7 & 9,4 & 9,5 & 9,4 & 9,4 \\
\hline TT g ácido tánico $/ \mathrm{kg}$ MS & & & & & & & 6,36 & 6,36 & 6,36 & 6,36 & 6,36 & 6,36 \\
\hline TC mg/500 mg MS & & & & & & & 2,10 & 2,10 & 2,10 & 2,10 & 2,10 & 2,10 \\
\hline \multicolumn{13}{|l|}{ Ácidos grasos (AG) } \\
\hline \multicolumn{13}{|c|}{ g de AG/100 g de AG totales } \\
\hline C12:0 & 0,9 & 0,9 & 1,0 & 0,7 & 0,9 & 0,8 & 0,9 & 0,7 & 0,8 & 0,6 & 0,8 & 0,7 \\
\hline C14:0 & 0,9 & 0,8 & 0,9 & 0,7 & 1,1 & 1,0 & 0,8 & 0,6 & 0,7 & 0,6 & 1,0 & 1,0 \\
\hline C16:0 & 23,9 & 20,6 & 23,5 & 19,5 & 37,1 & 39,4 & 23,1 & 19,4 & 21,3 & 18,1 & 37,2 & 40,2 \\
\hline C18:0 & 6,5 & 6,2 & 6,2 & 5,9 & 6,8 & 6,0 & 6,3 & 6,0 & 5,5 & 5,3 & 6,1 & 5,9 \\
\hline C18:1 c9 & 22,2 & 23,5 & 20,0 & 20,4 & 24,2 & 27,1 & 21,1 & 23,0 & 18,2 & 19,4 & 23,6 & 26,7 \\
\hline C18:1 t11 & 0,8 & 0,6 & 0,8 & 0,6 & 0,8 & 0,4 & 0,6 & 0,5 & 0,6 & 0,5 & 0,6 & 0,4 \\
\hline $\mathrm{C} 18: 2 \mathrm{c} 9,12$ & 33,6 & 37,4 & 22,7 & 20,7 & 18,3 & 17,8 & 33,5 & 37,7 & 21,8 & 20,6 & 18,2 & 15,8 \\
\hline C18:3 c9,12,15 & 5,4 & 4,5 & 19,2 & 26,8 & 6,0 & 4,0 & 7,9 & 6,5 & 26,0 & 30,4 & 7,5 & 5,6 \\
\hline \multicolumn{13}{|l|}{ Sumatorias } \\
\hline AG saturados & 35,7 & 31,7 & 34,9 & 29,3 & 48,6 & 49,4 & 34,5 & 29,9 & 31,2 & 27,1 & 48,1 & 50,1 \\
\hline AG monoinsaturados & 24,5 & 25,5 & 22,4 & 22,3 & 26,5 & 28,5 & 23,1 & 24,8 & 20,0 & 21,0 & 25,6 & 28,1 \\
\hline AG poliinsaturados & 39,9 & 42,9 & 42,9 & 48,4 & 25,0 & 22,1 & 42,6 & 45,3 & 48,8 & 51,8 & 26,6 & 21,9 \\
\hline
\end{tabular}

SSPi: sistema silvopastoril intensivo, FDN: fibra insoluble en detergente neutro, FDA: fibra insoluble en detergente ácido, TT: taninos totales, TC: taninos condensados / SSPi: intensive sylvopastoral system, FDN (NDF): neutral detergent fiber, FDA (ADF): acid detergent fiber, TT: total tannins, TC: condense tannins.

de fermentación, $5 \mathrm{ml}$ de líquido ruminal y $45 \mathrm{ml}$ de medio de cultivo, bajo un flujo de $\mathrm{CO}_{2}$. Las botellas se cerraron con tapones de caucho y se precintaron con cápsulas de aluminio; luego, las botellas se agitaron y se colocaron dentro de una estufa de cultivo que se mantuvo a $39^{\circ} \mathrm{C}$.
Se utilizaron dos frascos/inóculo que contenían medio de cultivo e inóculo, pero no sustrato, los cuales fueron usados como blancos para corregir la presión generada por la utilización de $\mathrm{CO}_{2}$ y la producida por la fermentación de los microorganismos presentes en el líquido ruminal. 
Cuadro 3. Composición nutricional y perfil de ácidos grasos de las dietas representativas de los sistemas doble propósito, con adición de diferentes aceites a nivel del $2 \%$ y $4 \%$ de la MS utilizadas en la evaluación in vitro antes de la incubación por veinticuatro horas. Medellín, Colombia. Agosto, 2013.

Table 3. Nutritional composition and fatty acid profile of the representative diets of dual-purpose systems with different oil addition to level of $2 \%$ and $4 \%$ of the DM used in the in vitro evaluation before incubation for twenty four hours. Medellin, Colombia. August, 2013.

\begin{tabular}{|c|c|c|c|c|c|c|c|c|c|c|c|c|}
\hline \multirow{4}{*}{$\begin{array}{c}\text { Dieta/Sistema } \\
\text { Aceite } \\
\text { Nivel } \\
\end{array}$} & \multicolumn{6}{|c|}{ Doble propósito } & \multicolumn{6}{|c|}{ Doble propósito SSPi } \\
\hline & \multicolumn{6}{|c|}{ Estrella $80 \%$ + concentrado $20 \%$} & \multicolumn{6}{|c|}{ Guinea $84 \%$ + leucaena $16 \%$} \\
\hline & \multicolumn{2}{|c|}{ Girasol } & \multicolumn{2}{|c|}{ Lino } & \multicolumn{2}{|c|}{ Palma } & \multicolumn{2}{|c|}{ Girasol } & \multicolumn{2}{|c|}{ Lino } & \multicolumn{2}{|c|}{ Palma } \\
\hline & $2 \%$ & $4 \%$ & $2 \%$ & $4 \%$ & $2 \%$ & $4 \%$ & $2 \%$ & $4 \%$ & $2 \%$ & $4 \%$ & $2 \%$ & $4 \%$ \\
\hline$\%$ grasa & 4,0 & 5,8 & 4,1 & 6,3 & 4,2 & 5,9 & 3,1 & 4,9 & 3,0 & 4,8 & 3,0 & 4,9 \\
\hline$\%$ proteína & 10,8 & 11,2 & 11,9 & 10,9 & 11,6 & 11,3 & 15,0 & 15,2 & 15,4 & 15,3 & 15,2 & 15,1 \\
\hline$\% \mathrm{FDN}$ & 65,5 & 65,0 & 65,5 & 64,1 & 64,5 & 63,5 & 59,2 & 52,3 & 59,5 & 58,5 & 59,1 & 59,0 \\
\hline$\%$ FDA & 30,1 & 29,0 & 29,8 & 29,4 & 29,6 & 29,2 & 29,3 & 26,1 & 29,7 & 29,5 & 29,1 & 29,4 \\
\hline$\%$ cenizas & 9,6 & 9,6 & 10,0 & 9,7 & 9,7 & 9,8 & 12,6 & 12,8 & 11,7 & 11,9 & 11,8 & 11,9 \\
\hline TT g ácido tánico/kg de MS & & & & & & & 6,8 & 6,8 & 6,8 & 6,8 & 6,8 & 6,8 \\
\hline TC mg/500 mg MS & & & & & & & 2,2 & 2,2 & 2,2 & 2,2 & 2,2 & 2,2 \\
\hline \multicolumn{13}{|l|}{ Ácidos grasos (AG) } \\
\hline \multicolumn{13}{|l|}{ g de AG/100 g de AG totales } \\
\hline C12:0 & 0,7 & 0,4 & 0,7 & 0,6 & 0,7 & 0,6 & 0,4 & 0,3 & 0,4 & 0,2 & 0,4 & 0,3 \\
\hline $\mathrm{C} 14: 0$ & 0,7 & 0,5 & 0,7 & 0,5 & 0,9 & 0,9 & 0,4 & 0,4 & 0,4 & 0,3 & 0,7 & 0,7 \\
\hline $\mathrm{C} 16: 0$ & 21,5 & 19,2 & 21,4 & 17,1 & 36,8 & 41,3 & 19,4 & 17,4 & 18,5 & 14,5 & 40,4 & 42,7 \\
\hline C18:0 & 5,4 & 5,7 & 5,3 & 4,9 & 5,2 & 5,4 & 4,2 & 5,0 & 3,7 & 3,9 & 4,2 & 4,4 \\
\hline C18:1 c9 & 21,2 & 23,7 & 18,1 & 19,4 & 23,9 & 27,2 & 16,5 & 21,7 & 13,4 & 16,6 & 21,8 & 26,5 \\
\hline C18:1 t11 & 0,7 & 0,5 & 0,6 & 0,4 & 0,5 & 0,4 & & 0,4 & & & 0,5 & \\
\hline C18:2 c9,12 & 35,8 & 35,5 & 20,5 & 20,6 & 17,5 & 15,0 & 34,9 & 36,7 & 16,9 & 17,2 & 11,4 & 10,6 \\
\hline C18:3 c9,12,15 & 8,2 & 5,6 & 25,4 & 32,3 & 6,8 & 5,3 & 15,7 & 10,1 & 39,8 & 41,2 & 14,0 & 9,1 \\
\hline \multicolumn{13}{|l|}{ Sumatorias } \\
\hline AG saturados & 31,8 & 29,4 & 31,4 & 25,4 & 46,3 & 50,6 & 28,5 & 26,6 & 26,0 & 21,0 & 48,4 & 50,2 \\
\hline AG monoinsaturados & 23,2 & 28,8 & 21,6 & 21,0 & 28,8 & 28,7 & 19,8 & 25,9 & 16,3 & 19,7 & 25,9 & 29,6 \\
\hline AG poliinsaturados & 45,1 & 42,1 & 47,1 & 53,7 & 25,0 & 20,9 & 51,7 & 47,7 & 57,7 & 59,5 & 26,3 & 20,3 \\
\hline
\end{tabular}

SSPi: sistema silvopastoril intensivo, FDN: fibra insoluble en detergente neutro, FDA: fibra insoluble en detergente ácido, TT: taninos totales, TC: taninos condensados / SSPi: intensive sylvopastoral system, FDN (NDF): neutral detergent fiber, FDA (ADF): acid detergent fiber, TT: total tannins, TC: condensed tannins.

Al cabo de 2, 4, 6, 8,10,12,15, 24, 30, 36, 48, 72 y 96 h de incubación, se midió la producción de gas a partir del aumento de presión en el espacio de la cabeza de los viales, utilizando un transductor digital acoplado a una aguja que se introducía a través de la tapa de caucho de los frascos. La presión se midió en libras por pulgada cuadrada (PSI). Para transformar los datos de presión (PSI) $(\mathrm{X})$ en volumen de gas $(\mathrm{ml})$ $(\mathrm{Y})$, se utilizó la ecuación $\mathrm{Y}=-0,1375+(5,1385 * \mathrm{X})+$ $\left(0,0777 * \mathrm{X}^{2}\right)$ propuesta por Posada et al. (2006).

Al finalizar la incubación, el contenido de las botellas se filtró usando crisoles (poro número 1) 
de peso conocido, se utilizó una bomba de vacío. El residuo recuperado se secó en horno $\left(65^{\circ} \mathrm{C}\right.$ por $\left.48 \mathrm{~h}\right)$, luego se pesó y se usó para calcular por gravimetría la degradabilidad de la MS a 96 h (García-González et al., 2008).

Para establecer la relación entre la cantidad de sustrato degradado (mg) y el volumen de gas producido (ml) se calculó el factor de partición (FP), que es considerado como un factor de eficiencia microbiana (Duque et al., 2009).

\section{Determinación de metano, $\mathrm{pH}$,ácidos grasos volátiles y perfil de ácidos grasos (ALC, ATV y AGCL)}

Para la determinación de metano, pH, ácidos grasos volátiles y perfil de ácidos grasos (ALC, ATV y AGCL) se utilizaron botellas de fermentación, similares a las descritas previamente, preparadas de manera simultánea y de la misma forma; sin embargo, en este caso, la fermentación solo se adelantó por un periodo total de veinticuatro horas. Al cabo de este tiempo, se midió el volumen de gas acumulado en el espacio de cabeza de la botella, utilizando un transductor digital, y mediante una jeringa se tomó una muestra de gas presente en la botella y se colocó dentro de un tubo de ensayo $(10 \mathrm{ml})$ con vacío. Posteriormente, en esta muestra de gas, se determinó la concentración de metano mediante cromatografía de gases; para ello, se tomaron $200 \mu 1$ de muestra, los cuales se inyectaron manualmente, con una jeringa de $1 \mathrm{ml}$, en un cromatógrafo de gases equipado con detector de ionización de llama (FID) y una columna empacada modelo GS-AL/KCl, de $50 \mathrm{~m}$ de largo, $0,53 \mathrm{~mm}$ de diámetro, con fase móvil de nitrógeno al $99,995 \%$ de pureza, flujo constante de $1 \mathrm{ml} / \mathrm{min}$ y programación del horno con isoterma a $80{ }^{\circ} \mathrm{C}$, durante cinco minutos, seguido de una temperatura postcorrida de $100{ }^{\circ} \mathrm{C}$ durante un minuto. El contenido de metano, se determinó mediante la generación de una curva de calibración obtenida diluyendo un estándar de metano de alta pureza $(99,99 \%)$ con $\mathrm{CO}_{2}$, procedimiento que se realizó siguiendo los lineamientos de López y Newbold (2007).

La producción de metano $(\mathrm{ml})$ fue calculada a partir del volumen total de gas $(\mathrm{ml})$ y la concentración de metano, esta se expresó por gramo de materia seca incubada. De acuerdo con el protocolo utilizado, se incubaron $0,5 \mathrm{~g}$ de materia seca.
Del mismo modo, se hizo determinación de la concentración de metano a 48 horas, la muestra provenía de las botellas que se incubaron por $96 \mathrm{~h}$; para esto, el gas que produjo cada botella a las 2,4 , $6,8,10,12,15,24,30,36$ y 48 h de incubación, se colectó en la respectiva medición de producción de gas, con una jeringa conectada junto con el transductor digital a una válvula de tres salidas. La primera salida fue conectada a una aguja que se insertó en el interior de la botella incubada, la segunda al transductor de presión y la tercera a la jeringa plástica que colectaba el gas. El gas colectado fue acumulado en una bolsa herméticamente cerrada, utilizando una para cada botella; posteriormente, se tomó una muestra del gas presente en esta bolsa y se colocó dentro de un tubo de ensayo $(10 \mathrm{ml})$ con vacío.

Luego de abrir las botellas incubadas por veinticuatro horas, se midió el $\mathrm{pH}$ con $\mathrm{pH}$-metro digital y se tomó una muestra de líquido sobrenadante de cada botella $(0,8 \mathrm{ml})$, para hacer la determinación de ácidos grasos volátiles (AGV); esta muestra fue congelada inmediatamente; posteriormente, se preparó para análisis de cromatografía de gases, para esto la muestra fue descongelada y agitada, se tomaron $800 \mu 1$ y se colocaron en un tubo eppendorf, se adicionaron $500 \mu 1$ de una solución con 20 g/l de ácido metafosfórico y 4 g/l de ácido crotónico (usado como estándar interno) en ácido clorhídrico $0,5 \mathrm{~N}$, se tapó y dejó por dos horas, al cabo de las cuales se centrifugó a 13000 rpm durante quince minutos; seguidamente se tomó $1 \mathrm{ml}$ de sobrenadante y se colocó en viales de $1,5 \mathrm{ml}$, los cuales fueron colocados en el automuestreador del cromatógrafo. Se usó un cromatógrafo equipado con detector de ionización de llama, con una columna capilar TR-FFAP de $30 \mathrm{~m} \times$ $0,53 \mathrm{~mm} \times 1 \mathrm{~m}$. Las condiciones de temperatura fueron $50{ }^{\circ} \mathrm{C}$ iniciales en la columna por cinco minutos, $225^{\circ} \mathrm{C}$ por diez minutos con una gradiente de $5^{\circ} \mathrm{C}$ por minuto. El gas portador fue nitrógeno con un flujo de $1 \mathrm{ml} / \mathrm{minu}-$ to; el volumen de inyección de $0,4 \mu 1$ a una temperatura de $225{ }^{\circ} \mathrm{C}$ en modo split 1:50.

La concentración de AGV (mmol/litro) fue calculada a partir de la concentración (ppm) determinada por cromatografía, y asumiendo una masa molar de 60,05 g/mol para el ácido acético, $74,08 \mathrm{~g} / \mathrm{mol}$ para propiónico, $88,11 \mathrm{~g} / \mathrm{mol}$ para ácido butírico e isobutírico y $102,13 \mathrm{~g} / \mathrm{mol}$ para los ácidos pentanoico e isopentanoico, seguidamente se calculó la proporción molar de cada AGV. 
El contenido que quedó en las botellas, se usó para el análisis de AG; para esto, se almacenó a $-20{ }^{\circ} \mathrm{C}$ hasta el momento de la extracción de los lípidos y su análisis por cromatografía de gases, siguiendo el procedimiento descrito anteriormente para AG de los alimentos.

\section{Análisis estadístico}

La cinética de producción de gas fue ajustada al modelo de France et al. (2000), correspondiente a $\mathrm{G}=$

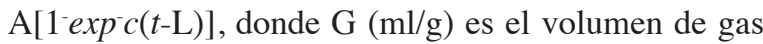
acumulado en el tiempo $(t) ; \mathrm{A}(\mathrm{ml} / \mathrm{g})$ es el volumen de gas correspondiente a la digestión completa del sustrato (asíntota); C (\%/hora) es la tasa constante de producción de gas y L (horas) es el tiempo de colonización. El ajuste de los datos al modelo y las estimativas de los parámetros se realizaron a través de PROC NLIN de SAS (2004).

El perfil de ácidos grasos (ALC-c9t11, ATV, AGCL), parámetros de producción de gas, FP, degradabilidad de la MS, producción de metano, $\mathrm{pH}$ y AGV, se analizaron mediante ANAVA en un diseño completamente al azar (CAA) con arreglo factorial de tratamientos $4 \times 3 \times 2$ (cuatro dietas $\mathrm{x}$ tres aceites $\mathrm{x}$ dos niveles de aceite). El efecto fijo en el modelo correspondió al tratamiento experimental y el efecto aleatorio al inóculo ruminal. La diferencia entre promedios se analizó mediante prueba de Tuckey con nivel de significancia del 5\%, utilizando PROC GLM de SAS (2004).

\section{RESULTADOS Y DISCUSIÓN}

\section{Composición de los tratamientos}

La composición química y ácidos grasos de la mezcla de los alimentos más el aceite antes de la incubación, indicó que en los tratamientos con adición de aceite de girasol, lino o palma, los AG más representativos fueron el linoleico, linolénico y palmítico, respectivamente, en todas las dietas en evaluación. No obstante, en los tratamientos con adición de aceite de lino, la participación del ácido linoleico y palmítico fue alta en dietas que incluían suplementación con concentrado, especialmente a nivel del $2 \%$ de adición de aceite (Cuadros 2 y 3 ).

\section{ALC-c9t11, ATV y otros AGCL en la fermen- tación ruminal}

La proporción de $\mathrm{AG}$ en la fermentación ruminal después de veinticuatro horas de incubación mostró efecto significativo del tipo de aceite $(p<0,0001)$, sobre el contenido de ALC-c9t11 (C18:2 c9t11) y ATV (C18:1 t11), siendo el aceite de girasol el que presentó los mayores porcentajes. No se presentó efecto significativo de la dieta, ni del nivel de inclusión de aceite $(p>0,05)$ sobre el contenido de ALC-c9t11 y ATV (Cuadro 4).

El aumento en la proporción de ALC-c9t11 y ATV después de veinticuatro horas de fermentación, con la suplementación con aceite de girasol en todas las dietas evaluadas, estuvo de acuerdo con lo reportado en estudios in vitro por Jacob et al. (2012), quienes compararon suplementación con aceite de girasol $(65,23 \%$ de ácido linoleico) vs aceite de soja $(51,65 \%$ de ácido linoleico) en dietas para ganado altas en fibra (relación forraje:concentrado de 65:35); también estuvieron de acuerdo con un estudio en el que se comparó el ácido oleico vs linoleico (Wu et al., 2013) y con otro estudio que comparó la mezcla de ácido linoleico y linolénico en diferentes proporciones en dieta a base de pasto (Castillo-Vargas, 2012), lo que confirma que los aceites de origen vegetal ricos en ácido linoleico (girasol, soja) son los más efectivos para aumentar ALC-c9t11 y ATV. La magnitud y los patrones de biohidrogenación (BHR) difieren con los diferentes aceites vegetales suplementados. El primer paso de la BHR a nivel ruminal de ácido linoleico es la isomerización a ALC-c9t11 y la posterior reducción a ATV, pero los primeros pasos de la BHR del linolénico no presentan este comportamiento (Jenkins et al., 2008; Lee y Jenkins, 2011). Por lo tanto, el mayor consumo de precursor (ácido linoleico) y las diferencias en el proceso de BHR, permiten explicar la mayor producción de ALC-c9t11 y ATV en los tratamientos con adición de aceite de girasol.

Dietas altas en forraje suplementadas con una pequeña cantidad de aceite de semillas con alto contenido de AG poliinsaturados produce principalmente ALC-c9t11 y ATV en la leche de vacas (Kraft et al., 2003; Cruz-Hernández et al., 2004; 2006). Las dietas evaluadas presentaron una proporción de pasto entre 65 y 100\%; sin embargo, no hubo diferencias entre dietas $(p>0,05)$ sobre el contenido de ALC-c9t11 y ATV después de la fermentación. 


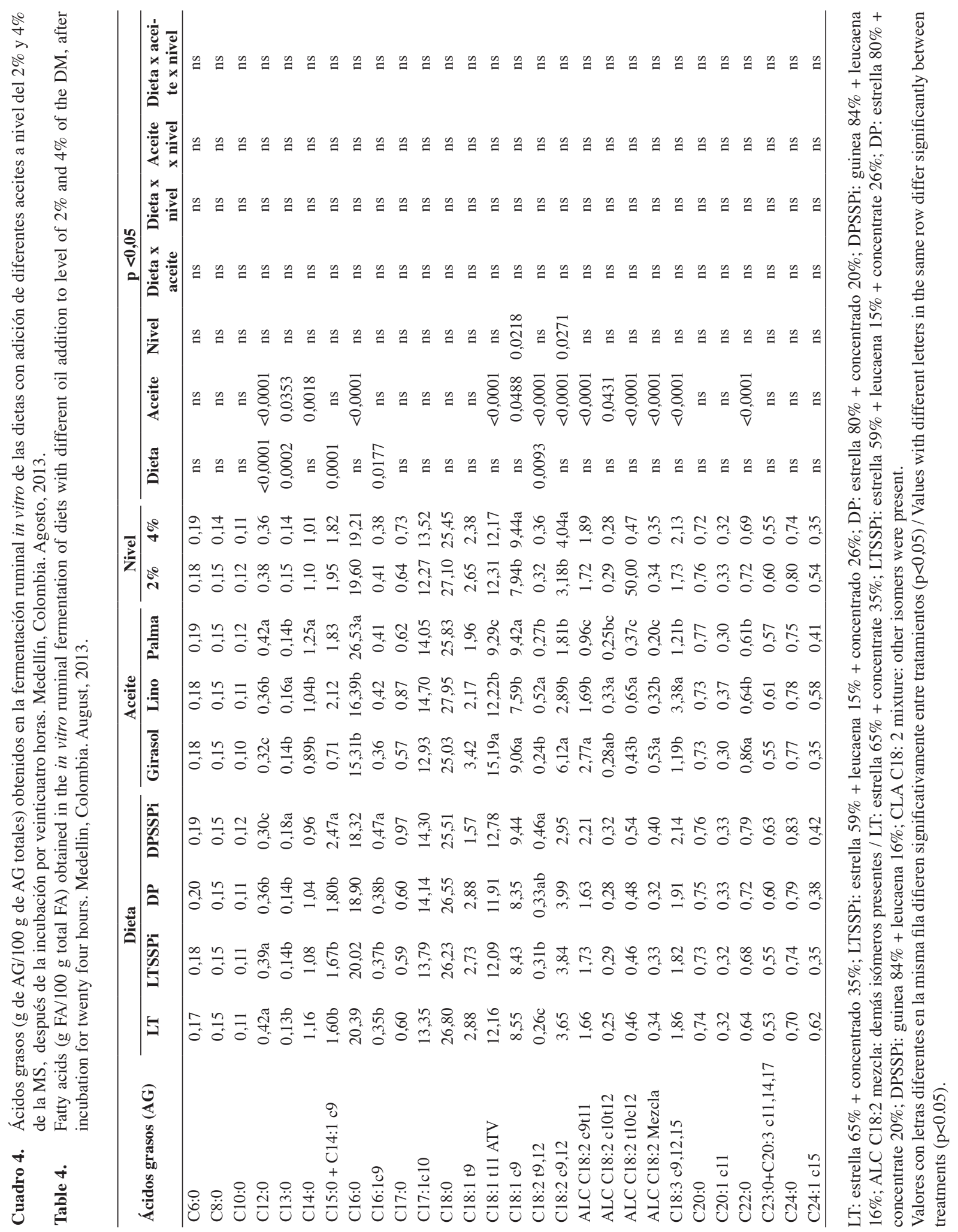


La suplementación con aceite de girasol aumentó el contenido de AG linoleico C18:2 c9,12 ( $p<0,0001)$, con un mayor valor a nivel de inclusión del $4 \%$ $(\mathrm{p}<0,02)$. También aumentó los ALC en mezcla $(p<0,0001)$ y el AG $C 22: 0(p<0,0001)$, mientras que el ALC t10c12 fue menor que con aceite de lino, sin efecto de la dieta, ni del nivel de inclusión de aceite. Asimismo, la suplementación con aceite de lino aumentó el contenido de AG linolénico C18:3 $\mathrm{c} 9,12,15(\mathrm{p}<0,0001)$, AG C18:2 t9,12 ( $<<0,0001)$, ALC c10t12 $(\mathrm{p}<0,04)$ y ALC t10c12 $(\mathrm{p}<0,0001)$. No se presentó efecto de la dieta, ni del nivel de inclusión de aceite sobre los AG linolénico, ALC c10t12 y ALC t10c12. Diferentes publicaciones (Harvatine y Bauman, 2006; Gervais et al., 2009) han mostrado que ALC t10c12 disminuye la grasa de la leche, a través de una baja regulación en la transcripción de las enzimas y proteínas que participan en la síntesis de lípidos en la glándula mamaria (Shingfield et al., 2010; Maxin et al., 2011). Los isómeros trans-10 y trans-11 son afectados por el ácido graso adicionado, mostrando que la producción de trans-10 fue mayor con adición de linoleico, mientras que linolénico fue principalmente hidrogenado vía trans-11 (Zened et al., 2011). Stoffel et al. (2015) reportaron un coeficiente positivo para el contenido de ácido linoleico en la dieta y $\mathrm{C} 18: 1 \mathrm{t} 10, \mathrm{ALC}$ t10c12 en la leche; no obstante, en el presente estudio la proporción de ALC t10c12 fue mayor para las dietas con adición de aceite de lino; en estos tratamientos la participación del ácido linoleico fue alta, especialmente en dietas que incluían suplementación con concentrado, lo que puedo implicar cambios en los procesos de la BHR, debidos al aumento en la cantidad total de ácidos linoleico y linolénico presentes en estas dietas.

La suplementación con aceite de palma aumentó el contenido de AG palmítico C16:0 ( $\mathrm{p}<0,0001)$, de AG mirístico C14:0 ( $<<0,0018)$, láurico $\mathrm{C} 12: 0$ $(\mathrm{p}<0,0001)$ y presentó el menor valor en los ALC evaluados, con respecto a aceite de girasol y lino. Dietas bajas en grasa $(1,2 \%)$ con adición de aceite de palma (1,7\% en base a MS), aumentaron los AG menores a C16:0 en la leche, y decrecieron el total de AG C18 comparado con adición de la misma cantidad de aceite rico en AG linoleico (Stoffel et al., 2015). La inclusión de grasas saturadas de origen animal en dietas para humanos puede incrementar el riesgo de enfermedades cardiovasculares (Joyce et al., 2009). Se ha demostrado que la suplementación con aceites vegetales ricos en $\mathrm{AG}$ poliinsaturados no solo permite aumentar los niveles de ALC-c9t11, sino que también aumenta ATV, AG insaturados (moni y poliinsaturados) y disminuye los AG saturados (Boerman y Lock 2014, Saliba et al., 2014; Vargas-Bello-Pérez et al., 2015), con un impacto alto en la composición de la grasa y su efecto sobre la salud humana. Lo anterior está de acuerdo con el presente trabajo, donde la adición de aceite de girasol y lino disminuyó C12:0, C14:0 y C16:0 con respecto a aceite de palma, y aumentó el total de AG C18 mono y poliinsaturados.

El AG oleico C18:1 c9, fue colectado por el tipo de aceite $(\mathrm{p}<0,0488)$ y el nivel de inclusión $(\mathrm{p}<0,0218)$, este fue menor cuando se suplementó con aceite de lino y mayor con un nivel del $4 \%$. La menor proporción de ácido oleico en los tratamientos con adición de aceite de lino después de la incubación, podría estar asociado a que estos tratamientos, antes de la incubación, presentaron la menor proporción de este ácido graso con respecto a los tratamientos con adición de aceite de girasol o palma; la mayor proporción de oleico con un nivel del $4 \%$, puede estar relacionada con la mayor concentración de grasa a este nivel, y a la alta proporción de este ácido graso en los aceites de girasol y palma.

El AG esteárico C18:0 fue similar entre dietas, fuente de aceite y nivel de inclusión $(p>0,05)$. Por lo tanto, la suplementación con aceites vegetales, la dieta y el nivel de inclusión de aceite, no afectaron la biohidrogenación completa; esta última se llevó a cabo en todos los tratamientos, alcanzándose valores de esteárico similares. Un nivel de aceite de girasol o soja al 6\% de la MS redujo el AG esteárico con respecto a un nivel del $4,5 \%$, posiblemente por una inhibición en la biohidrogenación con nivel del 6\% (Jacob et al., 2012). En este estudio con nivel de 2 y $4 \%$ no se afectó la biohidrogenación completa.

No hubo efecto de las interacciones entre dieta, fuente de aceite y nivel de aceite $(p>0,05)$ sobre los AG evaluados. Lo anterior muestra una respuesta independiente y clara de las diferentes fuentes de aceite y el nivel de inclusión sobre los AG evaluados.

\section{Parámetros de fermentación, degradabilidad y producción de metano}

La cinética de la fermentación, el pH, el total de AGV y la proporción molar de AGV no se vieron afectados por la fuente de aceite, ni el nivel de aceite 
( $\mathrm{p}>0,05)$, pero si por la dieta $(\mathrm{p}<0,0001)$ (Cuadros 5 y 6). La dieta que incluía estrella $80 \%$ + concentrado $20 \%$, presentó la mayor producción de gas (A) $(\mathrm{p}<0,0017)$. La dieta que incluía la mayor proporción de concentrado (estrella 65\% + concentrado 35\%) presentó una tasa mayor de producción de gas (c) $(\mathrm{p}<0,0001)$, y la dieta de solo forraje que no incluía concentrado (guinea $84 \%$ + leucaena 16\%), presentó el mayor tiempo de colonización (L) $(\mathrm{p}<0,0001)$. El total de AGV fue mayor para la dieta con mayor concentrado y menor para la dieta de solo forraje $(\mathrm{p}<0,0001)$. Igualmente, esta última presentó menor proporción de los ácidos propiónico, butírico, y mayor proporción de ácido acético y relación acético: propiónico que la primera $(\mathrm{p}<0,0001)$. Estos resultados están de acuerdo con los hallados por Jacob et al. (2012), quienes no encontraron efecto sobre $\mathrm{pH}$, total de $\mathrm{AGV}$, proporción de acético, propiónico, butírico y relación acético:propiónico, al suplementar con aceite de girasol o de soya a nivel del $4,5 \%$ de la MS, en dietas para ganado altas en fibra (relación forraje:concentrado 65:35), pero si se presentó un efecto significativo sobre estos parámetros con nivel del 6\% de la MS. Similares resultados se obtuvieron en un estudio en el que se suplementó con aceite de lino a nivel de 2,3 o 4\% de la MS, con una ración totalmente mezclada, con relación forraje:concentrado 50:50, donde no se encontró efecto sobre $\mathrm{pH}$, concentración total de $\mathrm{AGV}$, ni sobre el número total de protozoos (Benchaar et al., 2012). Sin embargo, Broudiscou et al. (1994), reportaron un decrecimiento en la concentración total de AGV en ovejas suplementadas con 6\% de aceite de lino, en dietas con relación forraje:concentrado 55:45. En general, se asume que la suplementación de grasas no protegidas y altamente insaturadas en dietas de rumiantes, disminuye la proporción de acetato y la relación acetato: propionato en el rumen, con un efecto antimicrobiano de los aceites ricos en ácidos grasos poliinsaturados como una probable explicación de este fenómeno (Jenkins y Jenny, 1992). No obstante, el tipo y fuente de aceite y la relación forraje:concentrado de la dieta basal, son los factores que determinan mayormente el efecto de la suplementación con lípidos sobre la fermentación ruminal (Toral et al., 2009). Las dietas utilizadas en el presente estudio presentaron una alta participación de forraje, lo que pudo favorecer la fermentación ruminal.

El porcentaje de degradabilidad de la MS fue afectado por la dieta, la fuente de aceite y su nivel de inclusión. La dieta de solo forraje de guinea $84 \%$ y leucaena $16 \%$, fue la que presentó la menor degradabilidad $(64,8 \%$ vs 69,$3 ; 69,7 ; 69,1)$ $(\mathrm{p}<0,0001)$. La suplementación con aceite de palma fue diferente, presentando la mayor degradabilidad (69\%) $(\mathrm{p}<0,0036)$. Los aceites de girasol y lino disminuyeron la degradabilidad en $2,3 \%$ y $1,5 \%$, respectivamente, con respecto a palma, siendo similares entre sí. El nivel de aceite del $4 \%$ presentó menor degradabilidad que el de $2 \%$ (66,5\% vs $69,9 \%$ ) $(\mathrm{p}<0,0001)$. Así mismo, el FP, considerado como un factor de eficiencia microbiana (Duque et al., 2009), fue afectado por el nivel de inclusión de aceite, este fue bajo con un nivel de inclusión de aceite de $4 \%$ $(\mathrm{p}<0,0415)$. Un aspecto importante a tener en cuenta en la alimentación de los animales rumiantes es que, debido al efecto inhibidor de los lípidos sobre el metabolismo microbiano, un aporte de alimentos con elevados contenidos en grasa puede provocar una inhibición de la fermentación ruminal, disminuyendo significativamente la digestibilidad y el consumo de alimento (Harfoot y Hazlewood, 1997). Generalmente, se recomienda que la grasa total no exceda del 6-7\% de la MS de la dieta, de otra forma puede ocurrir una depresión en el consumo de alimento (NRC, 2001). En este estudio, ocho de las doce dietas con adición de aceite al $4 \%$, estuvieron entre 6,40 y $7,62 \%$ de grasa, lo que pudo llevar a este resultado, y aunque se presentó una disminución en la degradabilidad con los aceites insaturados y a nivel del $4 \%$, esta se mantuvo por encima de $66,4 \%$.

No hubo efecto $(p>0,05)$ de la dieta, fuente de aceite, ni nivel de inclusión de aceite, sobre la producción de metano. Los aceites ricos en ácidos grasos de cadena media reducen la producción de metano (Machmüller, 2006), los ácidos láurico C12:0 y mirístico C14:0 han sido los más eficaces (Dohme et al., 2001; Soliva et al., 2003; Panyakaew et al., 2013). Igualmente, los ácidos grasos poliinsaturados de la dieta constituyen una de las alternativas dietarias más prometedoras para deprimir la metanogénesis (Martin et al., 2006; 2010; Cieslak et al., 2013; Patra, 2013; Wu et al., 2013). Aunque la cantidad de hidrógeno utilizado en el proceso de biohidrogenación es pequeña (1\%), en comparación con la cantidad de hidrógeno utilizada para reducir el dióxido de carbono para producir metano (48\%; Czerkawski, 1986), la reducción de metano in vitro, inducida por la adición de grasa a la dieta, puede alcanzar hasta un 50\% (Machmüller 


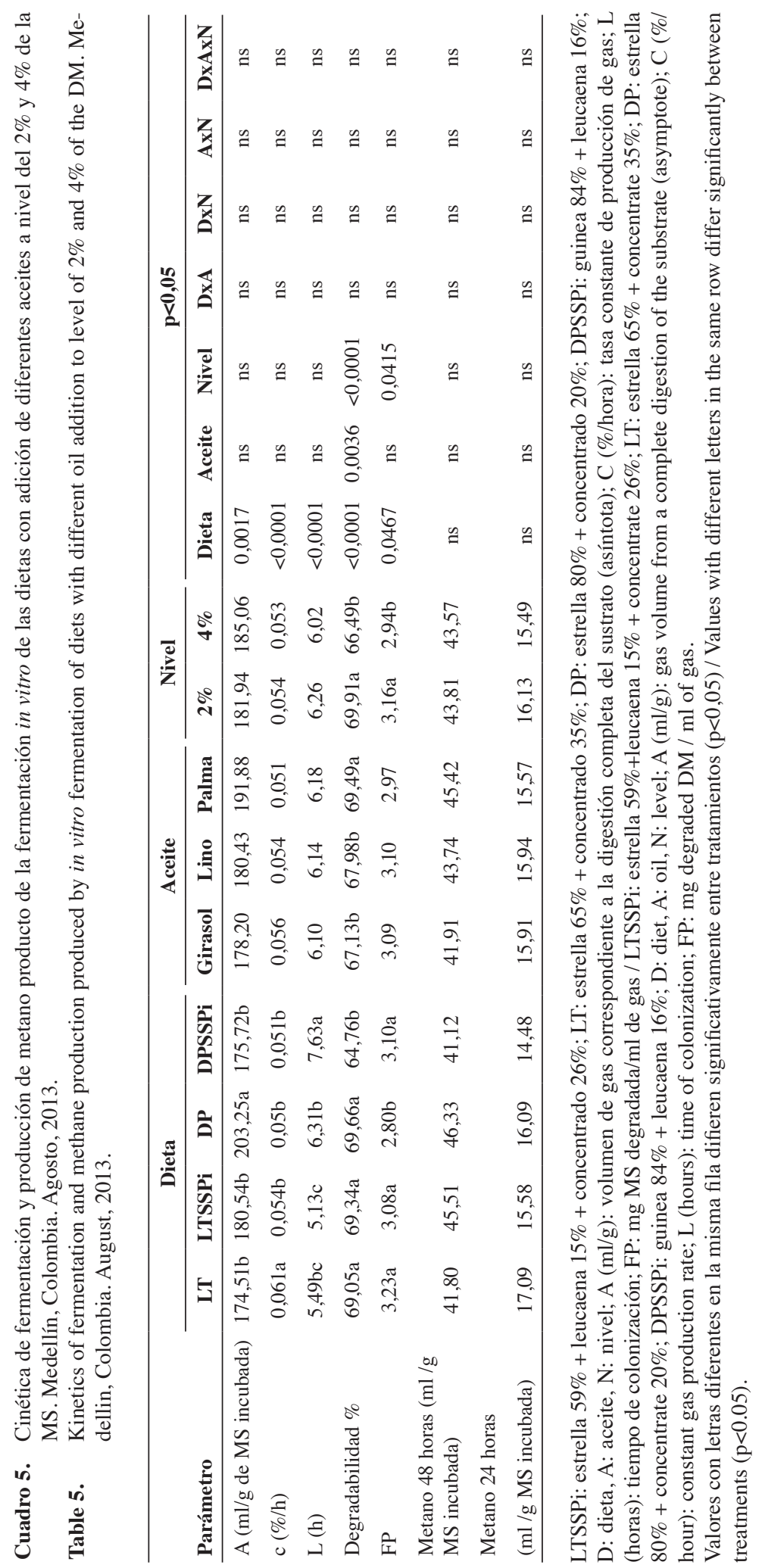


Cuadro 6. Concentración y proporción de ácidos grasos volátiles (AGV) producto de la fermentación in vitro de las dietas con adición de diferentes aceites a nivel del 2\% y 4\% de la MS. Medellín, Colombia. Agosto, 2013.

Table 6. Concentration and ratio of volatile fatty acids (VFA) produced by in vitro fermentation of diets with different oil addition to level of $2 \%$ and $4 \%$ of the DM. Medellin, Colombia. August, 2013.

\begin{tabular}{|c|c|c|c|c|c|c|c|c|c|c|c|c|c|c|c|c|}
\hline \multirow[b]{2}{*}{ Parámetro } & \multicolumn{4}{|c|}{ Dieta } & \multicolumn{3}{|c|}{ Aceite } & \multicolumn{2}{|l|}{ Nivel } & \multicolumn{7}{|c|}{$\mathrm{p}<0,05$} \\
\hline & LT & LTSSPi & DP & DPSSPi & $\begin{array}{c}\text { Gira- } \\
\text { sol }\end{array}$ & Lino & Palma & $2 \%$ & $4 \%$ & Dieta & $\begin{array}{c}\text { Acei- } \\
\text { te }\end{array}$ & $\begin{array}{l}\text { Ni- } \\
\text { vel }\end{array}$ & DxA & DxN & $\mathbf{A x N}$ & $\begin{array}{c}\text { DxAx } \\
\mathbf{N}\end{array}$ \\
\hline $\mathrm{pH}$ & $6,78 b$ & $6,78 b$ & $6,80 \mathrm{~b}$ & $6,85 \mathrm{a}$ & 6,81 & 6,79 & 6,81 & 6,80 & 6,81 & $<0,0001$ & $\mathrm{~ns}$ & $\mathrm{~ns}$ & ns & $\mathrm{ns}$ & ns & ns \\
\hline $\begin{array}{l}\text { Total AGV } \\
(\mathrm{mmol} / \mathrm{l})\end{array}$ & $29,66 \mathrm{a}$ & $28,75 \mathrm{ab}$ & $28,55 b$ & $26,77 \mathrm{c}$ & 28,14 & 28,36 & 28,71 & 28,77 & 28,14 & $<0,0001$ & ns & $\mathrm{ns}$ & ns & $\mathrm{ns}$ & ns & ns \\
\hline \multicolumn{17}{|c|}{ Proporción molar (\% molar) } \\
\hline Acético $\%$ & $61,84 \mathrm{c}$ & $62,89 b$ & $62,7 b$ & $66,04 \mathrm{a}$ & 63,26 & 63,35 & 63,49 & 63,45 & 63,28 & $<0,0001$ & ns & ns & ns & $\mathrm{ns}$ & ns & ns \\
\hline Propiónico\% & $29,18 \mathrm{a}$ & $28,79 \mathrm{ab}$ & $28,58 \mathrm{~b}$ & $26,58 \mathrm{c}$ & 28,35 & 28,43 & 28,07 & 28,22 & 28,34 & $<0,0001$ & ns & ns & ns & $\mathrm{ns}$ & ns & $\mathrm{ns}$ \\
\hline Butírico\% & $8,97 \mathrm{a}$ & $8,30 \mathrm{~b}$ & $8,70 \mathrm{ab}$ & $7,36 \mathrm{c}$ & 8,38 & 8,20 & 8,42 & 8,31 & 8,36 & $<0,0001$ & $\mathrm{~ns}$ & $\mathrm{~ns}$ & ns & $\mathrm{ns}$ & $\mathrm{ns}$ & ns \\
\hline $\begin{array}{l}\text { Acético: } \\
\text { propiónico }\end{array}$ & $2,12 \mathrm{c}$ & $2,18 \mathrm{~b}$ & $2,19 \mathrm{~b}$ & $2,48 \mathrm{a}$ & 2,23 & 2,23 & 2,26 & 2,25 & 2,24 & $<0,0001$ & ns & $\mathrm{ns}$ & ns & $\mathrm{ns}$ & ns & ns \\
\hline
\end{tabular}

LTSSPi: estrella 59\% + leucaena 15\% + concentrado 26\%; LT: estrella 65\% + concentrado 35\%; DP: estrella $80 \%$ + concentrado20\%; DPSSPi: guinea 84\% + leucaena 16\%; D: dieta, A: aceite, N: nivel / LTSSPi: estrella 59\% + leucaena 15\% + concentrate 26\%; LT: estrella 65\% + concentrate 35\%; DP: estrella 80\% + concentrate 20\%; DPSSPi: guinea 84\% + leucaena 16\%; D: diet, A: oil, N: level. Valores con letras diferentes en la misma fila difieren significativamente entre tratamientos $(\mathrm{p}<0,05) /$ Values with different letters in the same row differ significantly between treatments $(\mathrm{p}<0.05)$.

et al., 1998), y se ha observado que está asociada en gran parte a la disminución de protozoarios (Cieslak et al., 2006). Los metanógenos dependen de la actividad metabólica de los protozoos (Janssen, 2010). Así, el efecto supresor sobre producción de metano de C18:2 y $\mathrm{C} 18: 3$, puede ser debido a un efecto tóxico indirecto sobre los metanógenos del rumen.

En el presente estudio, la suplementación con diferentes aceites vegetales y el nivel de inclusión de aceite no afectaron producción de metano. La no diferencia entre aceites utilizados concuerda con lo reportado por Beauchemin et al. (2009) y Jalc et al. (2007), quienes encontraron un efecto similar sobre la producción de metano ruminal con los ácidos grasos C18: linoleico y linolénico. Sin embargo, es contraria a diversos estudios in vitro que sí han encontrado diferencias. Un estudio in vitro que evaluó el aceite de coco en dosis de 80 o $120 \mathrm{mg}$ de C12:0+C14:0 $/ 100 \mathrm{ml}$ de fluido de incubación, con y sin adición de $20 \mathrm{mg}$ de una mezcla de aceite de girasol y lino (GL), redujo la producción de metano en 12,7 y $14,5 \%$ para la dosis de 80 y $120 \mathrm{mg}$ sin adición de GL y en 31 y $28 \%$ para la dosis de 80 y $120 \mathrm{mg}$ con adición de GL (Panyakaew et al., 2013). Wu et al. (2013), adicionando $50 \mathrm{mg} / 500 \mathrm{mg}$ de sustrato de ácido oleico o linoleico, disminuyeron la producción de metano con ambos aceites. Cieslak et al. (2013) evaluaron la adición de $50 \mathrm{~g} / \mathrm{kg}$ de MS de aceite de uva (69,6\% de ácido linoleico) o aceite de grosella negra (58,6\% de ácido linoleico), y encontraron que la adición de los aceites no afectó la fermentación y se presentó una disminución en la producción de metano en 21 y $23 \%$ a las veinticuatro horas de fermentación para los tratamientos con aceite de uva y grosella, respectivamente. De otra parte, en un estudio donde se evaluó el ácido estearidónico (C18:4n3), a niveles de 1, 5,20 y $50 \mathrm{mg} / \mathrm{l}$ de medio de incubación, no se encontró efecto de la adición de aceite sobre la producción de metano, concluyendo que se necesitan altos niveles de ácido estearidónico no esterificado para mitigar metano (Amaro et al., 2012). En el presente estudio, la cantidad de aceite adicionada fue de $10 \mathrm{mg} / 500 \mathrm{mg}$ de sustrato o $20 \mathrm{mg} / 500 \mathrm{mg}$ de sustrato para el nivel de $2 \%$ o $4 \%$ de inclusión de aceite respectivamente, que corresponde con 20 o $40 \mathrm{~g}$ de aceite/kg de MS y con 5,59 o 11,19 mg de ácido linoleico/500 mg de sustrato 
para el nivel de $2 \%$ o $4 \%$, respectivamente, cuando se adicionó aceite de girasol (55,95\% de ácido linoleico), cantidades inferiores a las utilizadas en los trabajos de Cieslak et al. (2013) de $50 \mathrm{~g}$ de aceite/kg de MS y de Wu et al. (2013), de $50 \mathrm{mg}$ de ácido linoleico/500 $\mathrm{mg}$ de sustrato, lo que sugiere que la cantidad de aceite utilizada en este estudio no fue suficiente para disminuir metano.

No hubo efecto de las interacciones entre dieta, fuente de aceite y nivel de aceite $(\mathrm{p}>0,05)$, sobre las variables evaluadas.

La utilización de leucaena en el presente trabajo se hizo incorporando la cantidad de forraje representativo de dietas observadas en los animales en condiciones de campo (Prieto-Manrique, 2015). Las dietas de los sistemas silvopastoriles no fueron diferentes a las dietas de gramíneas solas en cuanto a la concentración de ALC-c9t11, ATV, esteárico o producción de metano, indicando que los taninos presentes en la leucaena no redujeron la BHR (biohidrogenación) o la producción de metano. Situación que pudo deberse a la baja concentración de taninos en las dietas evaluadas. En la dieta de la finca del sistema lechería tropical SSPi (LTSSPi), la inclusión de leucaena fue de 15\% y en la finca del sistema doble propósito SSPi (DPSSPi) fue del $16 \%$. La leucaena utilizada en el presente estudio, contenía $4,24 \%$ de taninos totales (\% de ácido tánico), lo que corresponde a 6,36 y $6,78 \mathrm{~g}$ de ácido tánico/kg de MS o $0,63 \%$ y $0,67 \%$ de la MS, para las fincas LTSSPi y DPSSPi, respectivamente. Igualmente, en el presente estudio las dietas de los sistemas LTSSPi y DPSSPi contenían 2,10 y $2,24 \mathrm{mg}$ de TC (taninos condensados)/500 $\mathrm{mg}$ de MS, respectivamente, que corresponden a $0,42 \%$ y $0,45 \%$ de la dieta, respectivamente. En estudios in vitro se reportó una disminución en la BHR de los ácidos linoleico y linolénico utilizando taninos de Quebracho, 22,3 g de ácido tánico/kg de MS, cantidad superior a la utilizada en el presente estudio (Minieri et al., 2014). Asimismo, Khiaosa-Ard et al. (2009) reportaron una inhibición del paso final de la BHR del ácido linolénico, utilizando 7,9\% de la MS de taninos condensados (TC), proporción superior a la utilizada en el presente estudio. Una reducción en la producción de metano fue mostrada por Tan et al. (2011), con inclusión de $10 \mathrm{mg}$ de TC/500 mg de MS; sin embargo, se ha demostrado que la producción de metano no disminuyó cuando $0,2 \%$ y $1,8 \%$ de taninos condensados fueron incorporados en la dieta (Sliwiński et al., 2002; Beauchemin et al., 2007). Por lo tanto, el bajo nivel de taninos utilizado en este estudio, pudo no haber afectado la producción de metano.

Es posible que el bajo nivel de aceite y de taninos empleado en las dietas en evaluación no haya afectado protozoos y metanógenos del rumen, así como tampoco se afectó el buen funcionamiento ruminal. No obstante, aunque no se logró disminuir la producción de metano, cuando se busca seleccionar el aceite y nivel de inclusión que presente mejor ALC-c 9 t11, ATV y AGCL mediante niveles prácticamente factibles de suplementación con aceite, el aceite de girasol a nivel del 2 y $4 \%$ puede ser una estrategia a evaluar bajo condiciones de campo, que permita aumentar los ácidos grasos benéficos en la leche de ganaderías colombianas manejadas con y sin sistemas silvopastoriles de leucaena con estrella y/o guinea.

\section{LITERATURA CITADA}

Amaro, P., M.R.G. Maia, R.J. Dewhurst, A.J.M. Fonseca, and A.R.J. Cabrita. 2012. Effects of increasing levels of stearidonic acid on methane production in a rumen in vitro system. Anim. Feed Sci. Technol. 173:252-260.

AOAC (Association of Official Analytical Chemists). 1999. Official methods of analysis of AOAC International. $16^{\text {th }}$ ed. AOAC International, Gaithersburg, MD, USA.

Beauchemin, K.A., M. Kreuzer, F. O’Mara, and T.A. McAllister. 2008. Nutritional management for enteric methane abatement: a review. Aust. J. Exp. Agric. 48(2):21-27.

Beauchemin, K.A., S.M. McGinn, C. Benchaar, and L. Holtshausen. 2009. Crushed sunflower, flax, or canola seeds in lactating dairy cow diets: effects on methane production, rumen fermentation, and milk production. J. Dairy Sci. 92:2118-2127.

Beauchemin, K.A., S.M. McGinn, T.F. Martinez, and T.A. McAllister. 2007. Use of condensed tannin extract from quebracho trees to reduce methane emissions from cattle. J. Anim. Sci. 85:1990-1996.

Bauman, D., and J. Griinari. 2001. Regulation and nutritional manipulation of milk fat: low-fat milk syndrome. Livest. Prod. Sci. 70:15-29.

Belury, M.A. 2002. Dietary conjugated linoleic acid in health: physiological effects and mechanisms of action. Annu. Rev. Nutr. 22:505-531.

Benchaar, C., G.A. Romero-Pérez, P.Y. Chouinard, F. Hassanat, M. Eugene, H.V. Petit, and C. Côrtes. 2012. Supplementation of increasing amounts of linseed 
oil to dairy cows fed total mixed rations: effects on digestion, ruminal fermentation characteristics, protozoal populations, and milk fatty acid composition. J. Dairy Sci. 95:4578-4590.

Bichi, E., P.G. Toral, G. Hervás, P. Frutos, P. Gómez-Cortés, M. Juárez, and M.A. De la Fuente. 2012. Inhibition of $\Delta$ 9-desaturase activity with sterculic acid: effect on the endogenous synthesis of cis-9 18:1 and cis-9, trans-11 18:2 in dairy sheep. J. Dairy Sci. 95:5242-5252.

Boerman, J.P., and A.L. Lock. 2014. Effect of unsaturated fatty acids and triglycerides from soybeans on milk fat synthesis and biohydrogenation intermediates in dairy cattle. J. Dairy Sci. 97:7031-7042.

Broudiscou, L., S. Pochet, and C. Poncet. 1994. Effect of linseed oil supplementation on feed degradation and microbial synthesis in the rumen of ciliate-free and refaunated sheep. Anim. Feed Sci. Technol. 49:189-202.

Castillo-Vargas, J.A. 2012. Cinética de biohidrogenación in vitro de ácidos grasos poliinsaturados en fluido ruminal. Tesis M.Sc., Universidad Nacional de Colombia, Bogotá, COL.

Chilliard, Y., A. Ferlay, J. Rouel, and G. Lamberet. 2003. A review of nutritional and physiological factors affecting goat milk lipid synthesis and lipolysis. J. Dairy Sci. 86:1751-1770.

Chilliard, Y., F. Glasser, A. Ferlay, L. Bernard, J. Rouel, and M. Doreau. 2007. Diet, rumen biohydrogenation and nutritional quality of cow and goat milk fat. Eur. J. Lipid Sci. Technol. 109:828-855.

Cieslak, A., M. Szumacher-Strabel, E. Szymankiewicz, M. Piękniewski, P. Oleszak, L. Siwiński, and A. Potkański. 2006. Coconut oil reduces protozoa count and methane release during fermentation in a Rusitec system. J. Anim. Feed Sci. 15:19-22.

Cieslak, A., Z. Varadyova, S. Kisidayova, D. Jalc, and M. Szumacher-Strabel. 2013. Effect of diets with fruit oils supplements on rumen fermentation parameters, fatty acid composition and methane production in vitro. $\mathrm{J}$. Anim. Feed Sci. 22:26-34.

CNL (Consejo Nacional Lácteo). 2010. Acuerdo de competitividad de la cadena láctea colombiana. CNL, Bogota, COL.

Collomb, M., H. Sollberger, U. Bütikofer, R. Sieber, W. Stoll, and W. Schaeren. 2004. Impact of a basal diet of hay and fodder beet supplemented with rapeseed, linseed and sunflowerseed on the fatty acid composition of milk fat. Int. Dairy J. 14:549-559.

CONPES (Consejo Nacional de Política Económica y Social). 2010. Política nacional para mejorar la competitividad del sector lácteo colombiano. Documento 3675. CONPES, Bogotá D.C., COL.

Cruz-Hernández, C., Z.Y. Deng, J.Q. Zhou, A.R. Hill, M.P. Yurawecz, P. Delmonte, M.M. Mossoba, M.E.R. Dugan, and J.K.G. Kramer. 2004. Methods for analysis of conjugated linoleic acids and trans-18:1 isomers in dairy fats by using a combination of gas chromatography, silverion thin-layer chromatography/gas chromatography, and silver-ion liquid chromatography. J. Assoc. Off. Anal. Chem. 87:545-562.

Cruz-Hernández, C., J.K.G. Kramer, J. Kraft, V. Santercole, M. Or-Rashid, D. ZeYuan, M.E.R. Dugan, P. Delmonte, M.P. Yurawecz, O. Gudmundsen, M.W. Pariza, and S. Banni. 2006. Systematic analysis of trans and conjugated linoleic acids in the milk and meat of ruminants. In: M.P. Yurawecz et al., editors, Advances in conjugated linoleic acid research. AOCS Press, Champaign, IL, USA. p. 45-93.

Czerkawski, J.W. 1986. An introduction to rumen studies. Oxford, Pergamon, TUR.

Dhiman, T.R., L.D. Satter, M.W. Pariza, M.P. Galli, K. Albright, and M.X. Tolosa. 2000. Conjugated linoleic acid (CLA) content of milk from cows offered diets rich in linoleic and linolenic acid. J. Dairy Sci. 83:1016-1027.

Dohme, F., A. Machmüller, A. Wasserfallen, and M. Kreuzer. 2001. Ruminal methanogenesis as influenced by individual fatty acids supplemented to complete ruminant diets. Lett. Appl. Microbiol. 32:47-51.

Duque, M., R. Noguera, y L.F. Restrepo. 2009. Efecto de la adición de urea protegida y sin protección sobre la cinética de degradación in vitro del pasto estrella (Cynodon nlemfluensis) y caña de azúcar (Saccharum officinarum). Livest. Res. Rural Dev. 21(4). http://www.lrrd.org/ lrrd21/4/duqu21058.htm (accesed 20 nov. 2014).

France, J., J. Dijkstra, M.S. Dhanoa, S. Lopez, and A. Bannink. 2000. Estimating the extent of degradation of ruminant feeds from a description of their gas production profiles observed in vitro: derivation of models and other mathematical considerations. Br. J. Nutr. 83:143-150.

García-González, R., S. López, M. Fernández, and J.S. González. 2008. Dose-response effects of Rheum officinale root and Frangulaalnus bark on ruminal methane production in vitro. Anim. Feed Sci. Technol. 145:319-334.

Gervais, R., J.W. McFadden, A.J. Lengi, B.A. Corl, and P.Y. Chouinard. 2009. Effects of intravenous infusion of t10, c12 18:2 on mammary lipid metabolism in lactating dairy cows. J. Dairy Sci. 92:5167-5177. 
Harfoot, C.G., and G.P. Hazlewood. 1997. Lipid metabolism in the rumen. In: P. Hobson, and C.S. Stewart, editors, The rumen microbial ecosystem. $2^{\text {nd }}$ ed. Blackie Academic and Professional Publishers, London, GBR. p. 382-426.

Harris, W.S. 2008. Linoleic acid and coronary heart disease. PLEFA 79:169-171.

Harvatine, K.J., and D.E. Bauman. 2006. SREBP1 and thyroid hormone responsive spot 14 (S14) are involved in the regulation of bovine mammary lipid synthesis during diet-induced milk fat depression and treatment with CLA. J. Nutr. 136:2468-2474.

Hervás, G., P. Luna, A.R. Mantecon, N. Castañares, P. Frutos, M.A. De la Fuente, and M. Juárez. 2006. Effect of sunflower oil on sheep milk production and composition, and in vitro rumen fermentation. In: European Federation for the Science and Technology of Lipids, editor, $4^{\text {th }}$ Euro Fed Lipid Congress, "Fats, oils and lipids for a healthier future". German Chemical Society (GDCh), Madrid, ESP. p. 571.

IPCC. 2007. Climate Change 2007: The physical science basis. In: S. Solomon et al., editors, Contribution of working group I to the fourth assessment report of the intergovernmental panel on climate change. Cambridge University Press, Cambridge, GBR and NY, USA.

Jacob, A.B., V. Balakrishnan, and C. Kathirvelan. 2012. Effect of amount and source of vegetable oils in a high fibrous cattle diet on in vitro rumen fermentation, nutrient degradability and rumen cis-9, trans-11 CLA concentration. J. Appl. Anim. Res. 40:148-153.

Jalc, D., M. Certik, K. Kundrikova, and P. Namestkova. 2007. Effect of unsaturated C18 fatty acids (oleic, linoleic and alpha-linolenic acid) on ruminal fermentation and production of fatty acid isomers in an artificial rumen. Vet. Med. - Czech 52(3):87-94.

Janssen, P. 2010. Influence of hydrogen on rumen methane formation and fermentation balances through microbial growth kinetics and fermentation thermodynamics. Anim. Feed Sci. Technol. 160:1-22.

Jayanegara, A., E. Wina, C.R. Soliva, S. Marquardt, M. Kreuzer, and F. Leiber. 2011. Dependence of forage quality and methanogenic potential of tropical plants on their phenolic fractions as determined by principal component analysis. Anim. Feed Sci. Technol. 163: 231-243.

Jenkins, T.C., and B.F. Jenny. 1992. Nutrient digestion and lactation performance of dairy cows fed combinations of prilled fat and canola oil. J. Dairy Sci. 75:796-803.
Jenkins, T.C., R.J. Wallace, P.J. Moate, and E.E. Mosley. 2008. Board-invited review: recent advances in biohydrogenation of unsaturated fatty acids within the rumen microbial ecosystem. J. Anim. Sci. 86:397-412.

Johnson, K.A., and D.E. Johnson. 1995. Methane emissions from cattle. J. Anim. Sci. 73:2483-2492.

Joyce, T., A.J. Wallace, S.N. McCarthy, and M.J. Gibney. 2009. Intakes of total fat, saturated, monounsaturated and polyunsaturated fatty acids in Irish children, teenagers and adults. Public Health Nutr. 12:156-165.

Kelly, M.L., J.R. Berry, D.A. Dwyer, J.M. Griinari, P.Y. Chouinard, M.E. Van Amburgh, and D.E. Bauman. 1998. Dietary fatty acid sources affect conjugated linoleic acid concentrations in milk from lactating dairy cows. J. Nutr. 128:881-885.

Khanal, R.C. 2004. Potential health benefits of conjugated linoleic acid (CLA): a review. Asian Australas. J. Anim. Sci. 17:1315-1328.

Khanal, R.C., and K.C. Olson. 2004. Factors affecting conjugated linoleic acid (CLA) content in milk, meat, and egg: A review. Pakistan J. Nutr. 3:2-98.

Khiaosa-Ard, R., S.F. Bryner, M.R.L. Scheeder, H.R. Wettstein, F. Leiber, M. Kreuzer, and C.R. Soliva. 2009. Evidence for the inhibition of the terminal step of ruminal alpha-linolenic acid biohydrogenation by condensed tannins. J. Dairy Sci. 92:177-188.

Kraft, J., M. Collomb, P. Möckel, R. Sieber, and G. Jahreis. 2003. Differences in CLA isomer distribution of cow's milk lipids. Lipids 38:657-664.

Lassey, K.R., M.J. Ulyatt, R.J. Martin, C.F. Walker, and I.D. Shelton. 1997. Methane emissions measured directly from grazing livestock in New Zealand. Atmos. Environ. 31:2905-2914.

Lee, Y.J., and T.C. Jenkins. 2011. Biohydrogenation of linolenic acid to stearic acid by the rumen microbial population yields multiple intermediate conjugated diene isomers. J. Nutr. 141:1445-1450.

Lock, A.L., and P.C. Garnsworthy. 2002. Independent effects of dietary linoleic and linolenic fatty acids on the conjugated linoleic acid content of cows' milk. Anim. Sci. 74:163-176.

López, S., and C.J. Newbold. 2007. Analysis of methane. In: H.P.S. Makkar, and P.E. Vercoe, editors, Measuring Methane Production from Ruminants. IAEA, FAO, Springer, Dordrecht, HOL. p. 1-10.

Machmüller, A. 2006. Medium-chain fatty acids and their potential to reduce methanogenesis in domestic ruminants. Agric. Ecosyst. Environ. 112:107-114. 
Machmüller, A., D.A. Ossowski, M. Wanner, and M. Kreuzer. 1998. Potential of various fatty feeds to reduce methane release from rumen fermentation in vitro (Rusitec). Anim. Feed Sci. Technol. 71:117-130.

Mahecha L., C.V. Durán, y M. Rosales. 2000. Análisis de la relación planta-animal desde el punto de vista nutricional en un sistema silvopastoril de Cynodon plectostachyus, Leucaena leucocephala y Prosopis juliflora en el Valle del Cauca. Acta Agronómica 50(1):59-70.

Makkar, H.P.S. 2003. Quantification of tannins in tree and shrub foliage. A laboratory manual. KluwerAcademic Publishers, HOL.

Martin, C., D.P. Morgavi, and M. Doreau. 2010. Methane mitigation in ruminants: from microbe to the farm scale. Anim. 4:351-365.

Martin, C., D.P. Morgavi, M. Doreau, and J.P. Jouany. 2006. Comment réduire la production de méthane chez les ruminants? Fourrages 187:283-300.

Maxin, G., H. Rulquin, and F. Glasser. 2011. Response of milk fat concentration and yield to nutrient supply in dairy cows. Anim. 5:1299-1310.

McDougall, E.I. 1948. Studies on ruminant saliva. 1. The composition and output of sheep's saliva. Biochem. J. 43:99-109.

Menke, K.H., and H. Steingass. 1988. Estimation of the energetic feed value obtained from chemical analysis and in vitro gas production using rumen fluid. Anim. Res. 28:7-55.

Milner, JA. 1999. Functional foods and health promotion. J. Nutr. 129:1395-1397.

Minieri, S., A. Buccioni, S. Rapaccini, A. Pezzati, D. Benvenuti, A. Serra, and M. Mele. 2014. Effect of Quebracho tannin extract on soybean and linseed oil biohydrogenation by solid associated bacteria: an in vitro study. Ital. J. Anim. Sci. 13:604-608.

Moss, A.R., J.P. Jouany, and J. Newbold. 2000. Methane production by ruminants: its contribution to global warming. Ann. Zootech. 49:231-253.

Murgueitio, E. 1999. Environmental and social adjustment of the cattle farming sector in Colombia. In: FAO, editor, World animal review. FAO, Roma, ITA. p. 2-15.

NRC (National Research Council). 2001. Nutrient requirements of dairy cattle. $7^{\text {th }}$ rev. National Academy Press, WA, USA.

Pagiola, S., P. Agostini, J. Gobbi, C. de Haan, M. Ibrahim, E. Murgueitio, E. Ramírez, M. Rosales, and J.P. Ruíz. 2005. Paying for biodiversity conservation services Experience in Colombia, Costa Rica, and Nicaragua. Mt. Res. Dev. 25:206-211.
Pagiola, S., E. Ramirez, J. Gobbi, C. De Haan, M. Ibrahim, E. Murgueitio, and J.P. Ruiz. 2007. Paying for the environmental services of silvopastoral practices in Nicaragua. Ecol. Econ. 64:374-385.

Palmquist, D.L. 2007. Biohydrogenation then and now. Eur. J. Lipid Sci. Technol. 109:737-739.

Palmquist, D.L., A.L. Lock, K.J. Shingfield, and D.E. Bauman. 2005. Biosynthesis of conjugated linoleic acid in ruminants and humans. Adv. Food Nutr. Res. 50:179-217.

Panyakaew, P., G. Goel, M. Lourenço, C. Yuangklang, and V. Fievez. 2013. Medium-chain fatty acids from coconut or krabok oil inhibit in vitro rumen methanogenesis and conversion of non-conjugated dienoic biohydrogenation intermediates. Anim. Feed Sci. Technol. 180:18-25.

Pariza, M.W. 2004. Perspective on the safety and effectiveness of conjugated linoleic acid. Am. J. Clinical Nutr. 79:1132-1136.

Patra, A.K. 2013. The effect of dietary fats on methane emissions, and its other effects on digestibility, rumen fermentation and lactation performance in cattle: Ameta-analysis. Livest. Sci. 155:244-254.

Posada, S., R. Noguera, y D. Bolívar. 2006. Relación entre presión y volumen para la implementación de la técnica in vitro de producción de gases. Rev. Colomb. Cienc. Pecu. 19:407-414.

Prieto-Manrique, E. 2015. Efecto de la suplementación con aceites vegetales a vacas pastoreando con/sin sistema silvopastoril intensivo con leucaena sobre los ácidos grasos en la leche y la producción de metano in vitro. Tesis PhD., Universidad de Antioquia, COL.

Saliba, L., R. Gervais, Y. Lebeuf, and P.Y. Chouinard. 2014. Effect of feeding linseed oil in diets differing in forage to concentrate ratio: 1 . Production performance and milk fat content of biohydrogenation intermediates of $\alpha$-linolenic acid. J. Dairy Res. 81:82-90.

SAS (Statistical Analysis System). 2004.Version 9.1.3. SAS Institute Inc., Cary, NC, USA.

Sheehle, E.A., and D. Kruger. 2006. Global anthropogenic methane and nitrous oxide emissions. The Energy J. 27 (special issue):33-44.

Shingfield, K.J., L. Bernard, C. Leroux, and Y. Chilliard. 2010. Role of trans fatty acids in the nutritional regulation of mammary lipogenesis in ruminants. Anim. 4:1140-1166.

Shingfield, K.J., Y. Chilliard, V. Toivonen, P. Kairenius, and D.I. Givens. 2008. Trans fatty acids and bioactive lipids in ruminant milk. Adv. Exp. Med. Biol. 606:3-65. 
Shingfield, K.J., C.K. Reynolds, G. Hervás, J.M. Griinari, A. S. Grandison, and D.E. Beever. 2006. Examination of the persistency of milk fatty acid composition responses to fish oil and sunflower oil in the diet of dairy cows. J. Dairy Sci. 89:714-732.

Sliwiński, B.J., M. Kreuzer, H.R. Wettstein, and A. Machmüller. 2002. Rumen fermentation and nitrogen balance of lambs fed diets containing plant extracts rich in tannins and saponins, and associated emissions of nitrogen and methane. Arch. Anim. Nutr. 56:379-392.

Soliva, C.R., I.K. Hindrichsen, L. Meile, M. Kreuzer, and A. Machmüller. 2003. Effects of mixtures of lauric and myristic acid on rumen methanogens and methanogenesis in vitro. Lett. Appl. Microbiol. 37:35-39.

Stanton, C., J. Murphy, E. McGrath, R. Devery, J.L. Sébédio, W.W. Christie, and R. Adlof. 2003. Animal feeding strategies for conjugated linoleic acid enrichment of milk. In: J.L. Sébédio et al., editors, Advances in conjugated linoleic acid research. AOCS Press, Champaign, IL, USA. p. 123-145.

Stoffel, C.M., P.M. Crump, and L.E. Armentano. 2015. Effect of dietary fatty acid supplements, varying in fatty acid composition, on milk fat secretion in dairy cattle fed diets supplemented to less than $3 \%$ total fatty acids. J. Dairy Sci. 98:431-442.

Tan, H.Y., C.C. Sieo, N. Abdullah, J.B. Liang, X.D. Huang, and Y.W. Ho. 2011. Effects of condensed tannins from Leucaena on methane production, rumen fermentation and populations of methanogens and protozoa in vitro. Anim. Feed Sci. Technol. 169:185-193.

Tequin-Ocampo, E. 2014. Estudio de la influencia de la suplementación lipídica en la dieta de bovinos sobre los ácidos grasos funcionales de la leche, y la producción de metano y ácidos grasos volátiles del fluido ruminal por cromatografía de gases. Tesis M.Sc., Universidad de Caldas, COL.

Theodorou, M.K., B.A. Williams, M.S. Dhanoa, A.B. McAllan, and J. France. 1994. A simple gas production method using a pressure transducer to determine the fermentation kinetics of ruminant feeds. Anim. Feed Sci. Technol. 48:185-197.

Toral, P., A. Belenguer, P. Frutos, and G. Hervás. 2009. Effect of the supplementation of a high-concentrate diet with sunflower and fish oils on ruminal fermentation in sheep. Small Rumin. Res. 81:119-125.

Van Soest, P.J., J.B. Robertson, and B.A. Lewis. 1991. Methods for dietary fiber, neutral detergent fiber, and nonstarch polysaccharides in relation to animal nutrition. J. Dairy Sci. 74:3583-3597.

Vargas-Bello-Pérez, E., K. Fehrmann-Cartes, G. ÍñiguezGonzález, P. Toro-Mujica, and P.C. Garnsworthy. 2015. Short communication: Chemical composition, fatty acid composition, and sensory characteristics of Chanco cheese from dairy cows supplemented with soybean and hydrogenated vegetable oils. J. Dairy Sci. 98:111-117.

Vasta, V., H.P.S. Makkar, M. Mele, and A. Priolo. 2009. Ruminal biohydrogenation as affected by tannins in vitro. Br. J. Nutr. 102:82-92.

Weiss, M.F., F.A. Martz, and C.L. Lorenzen. 2004a. Conjugated linoleic acid: historical context and implications. Prof. Anim. Sci. 20:118-126.

Weiss, M.F., F.A. Martz, and C.L. Lorenzen. 2004b. Conjugated linoleic acid: implicated mechanisms related to cancer, atherosclerosis, and obesity. Prof. Anim. Sci. 20:127-135.

Wu, D., S. Tang, Z. He, E.N. Odongo, Z. Tan, X. Han, C. Zhou, J. Kang, and M. Wang. 2013. Oleic and linoleic acids alter fermentation characteristics, methane and fatty acid isomers production during in vitro incubation with mixed ruminal microbes. J. Food Agric. Environ. 11:464-469.

Zened, A., A. Troegeler-Meynadier, M.C. Nicot, S. Combes, L. Cauquil, Y. Farizon, and F. Enjalbert. 2011. Starch and oil in the donor cow diet and starch in substrate differently affect the in vitro ruminal biohydrogenation of linoleic and linolenic acids. J. Dairy Sci. 94:5634-5645. 